\title{
Ring-degenerate rearrangement resulting from the azo coupling reaction of
}

\section{a 3-aryl-1,3a,6a-triazapentalene}

Yingchun Wang, † $^{\mathrm{a}}$ Tomas Opsomer, † $^{\mathrm{a}}$ Luc Van Meervelt ${ }^{\mathrm{b}}$ and Wim Dehaen $* a$

† These authors contributed equally to this work.

${ }^{a}$ Molecular Design and Synthesis, Department of Chemistry, KU Leuven, Celestijnenlaan 200F, 3001 Leuven, Belgium. E-mail: wim.dehaen@kuleuven.be.

${ }^{b}$ Biochemistry, Molecular and Structural Biology Section, Department of Chemistry, KU Leuven, Celestijnenlaan 200F, 3001 Leuven, Belgium

\section{Contents}

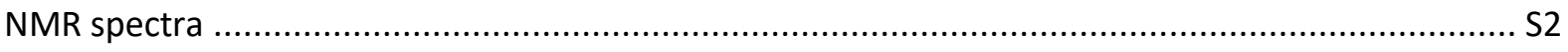

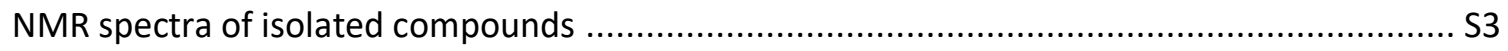

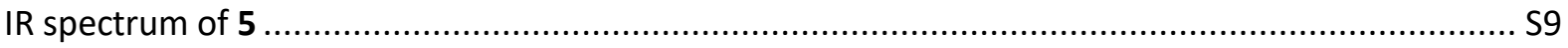

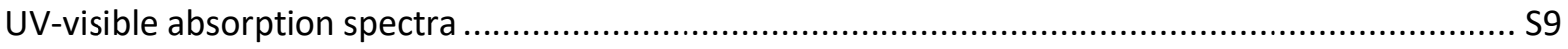

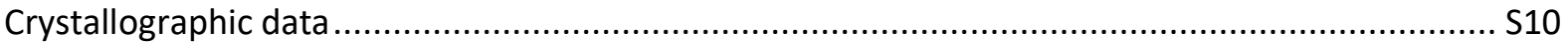

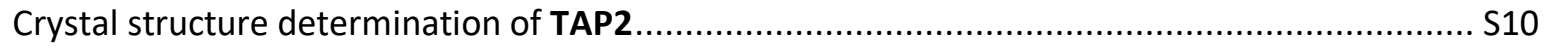

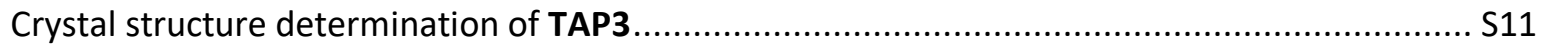

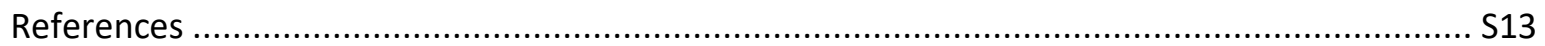




\section{NMR spectra}

A: TAP1

$\underbrace{\substack{n \\ \infty}}$

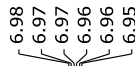

(isolated)

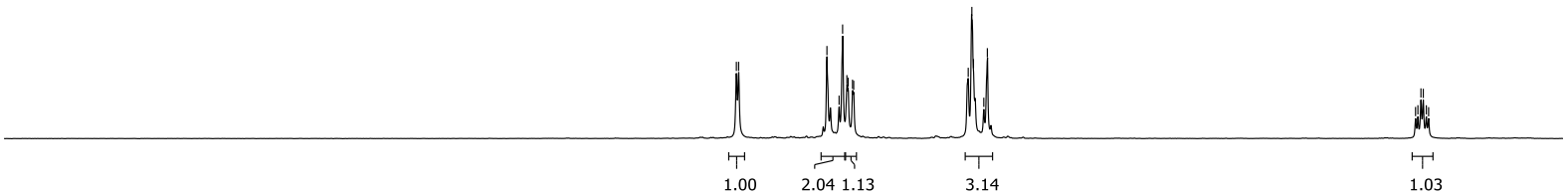

B: Crude mixture

(TAP1 + benzene

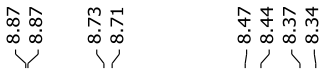

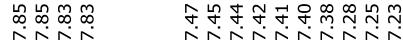

diazonium salt)

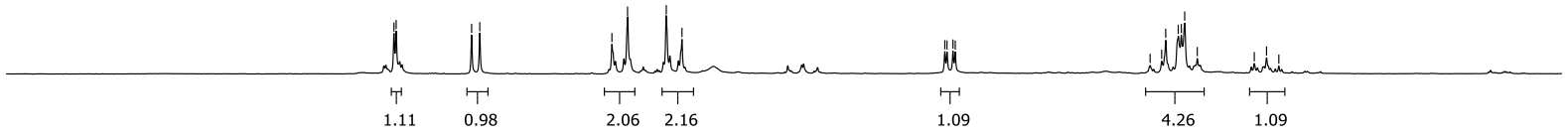

$\begin{array}{ll}\text { C: TAP3 } & \stackrel{2}{~} \text { (isolated) }\end{array}$

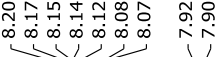

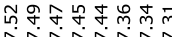

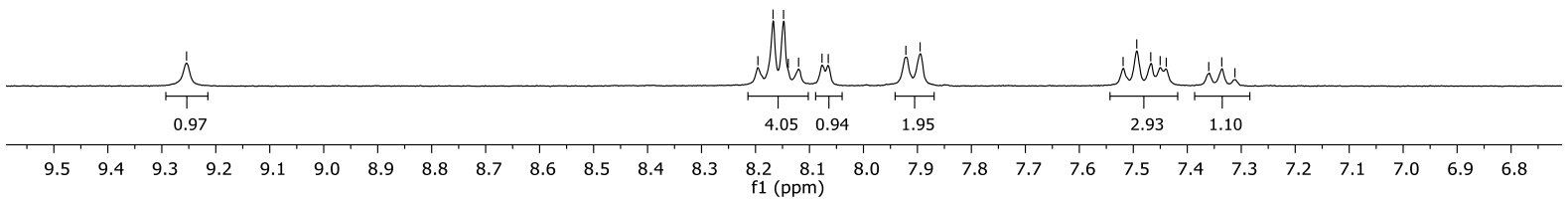

Figure S1. ${ }^{1} \mathrm{H}$ NMR spectra in acetone- $d_{6}(300 \mathrm{MHz})$ of A) TAP1, B) the crude reaction mixture of the azo coupling of TAP1 with benzenediazonium tetrafluoroborate and C) TAP3. The spectrum of the crude reaction mixture was measured within 3 minutes after the addition of the diazonium salt. No base was present in the reaction mixture. 
NMR spectra of isolated compounds

TAP2, ${ }^{1} \mathrm{H}, 300 \mathrm{MHz}, \mathrm{CDCl}_{3}$

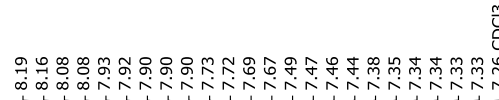

范
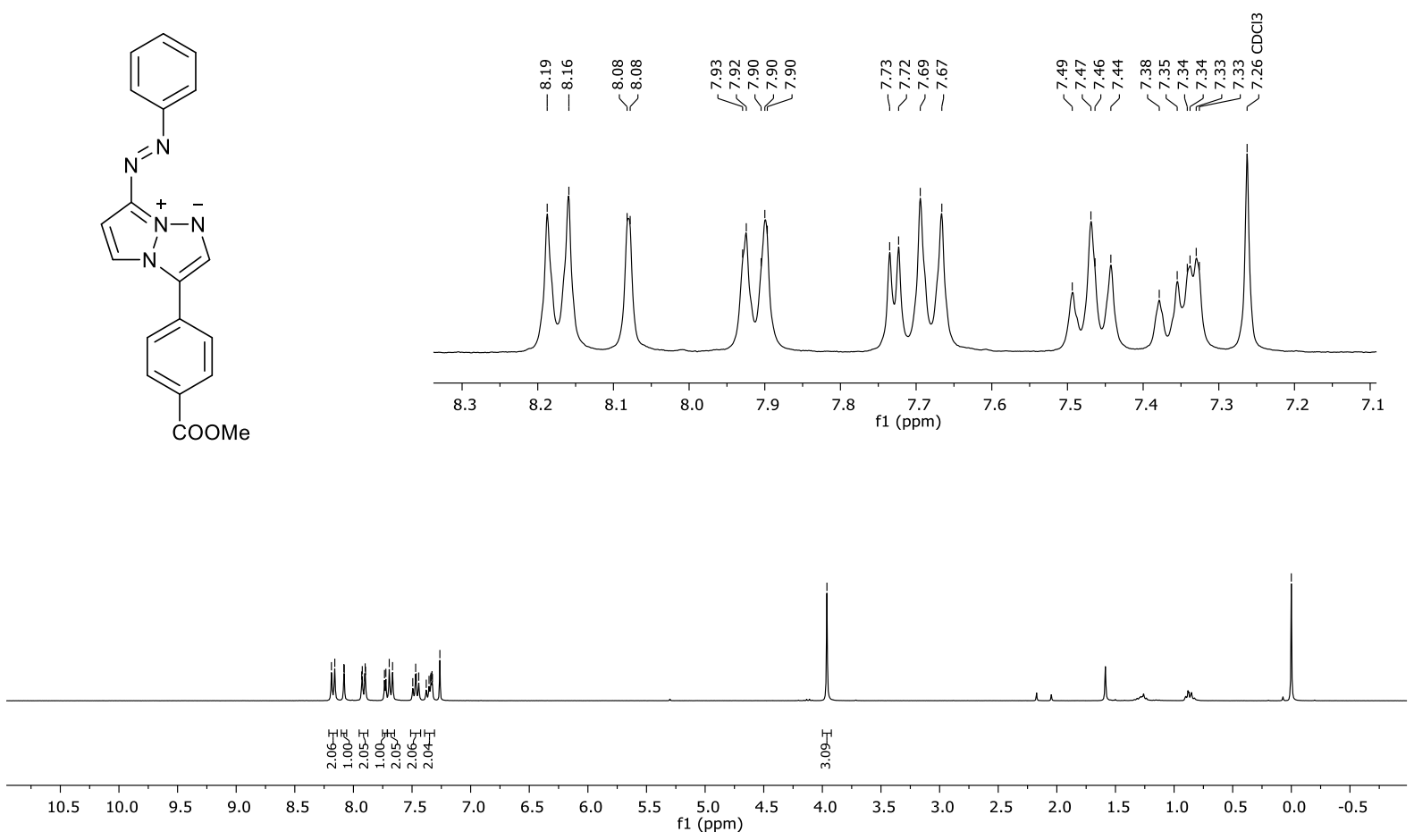

TAP2 $,{ }^{13} \mathrm{C}\left\{{ }^{1} \mathrm{H}\right\}, 151 \mathrm{MHz}, \mathrm{CDCl}_{3}$
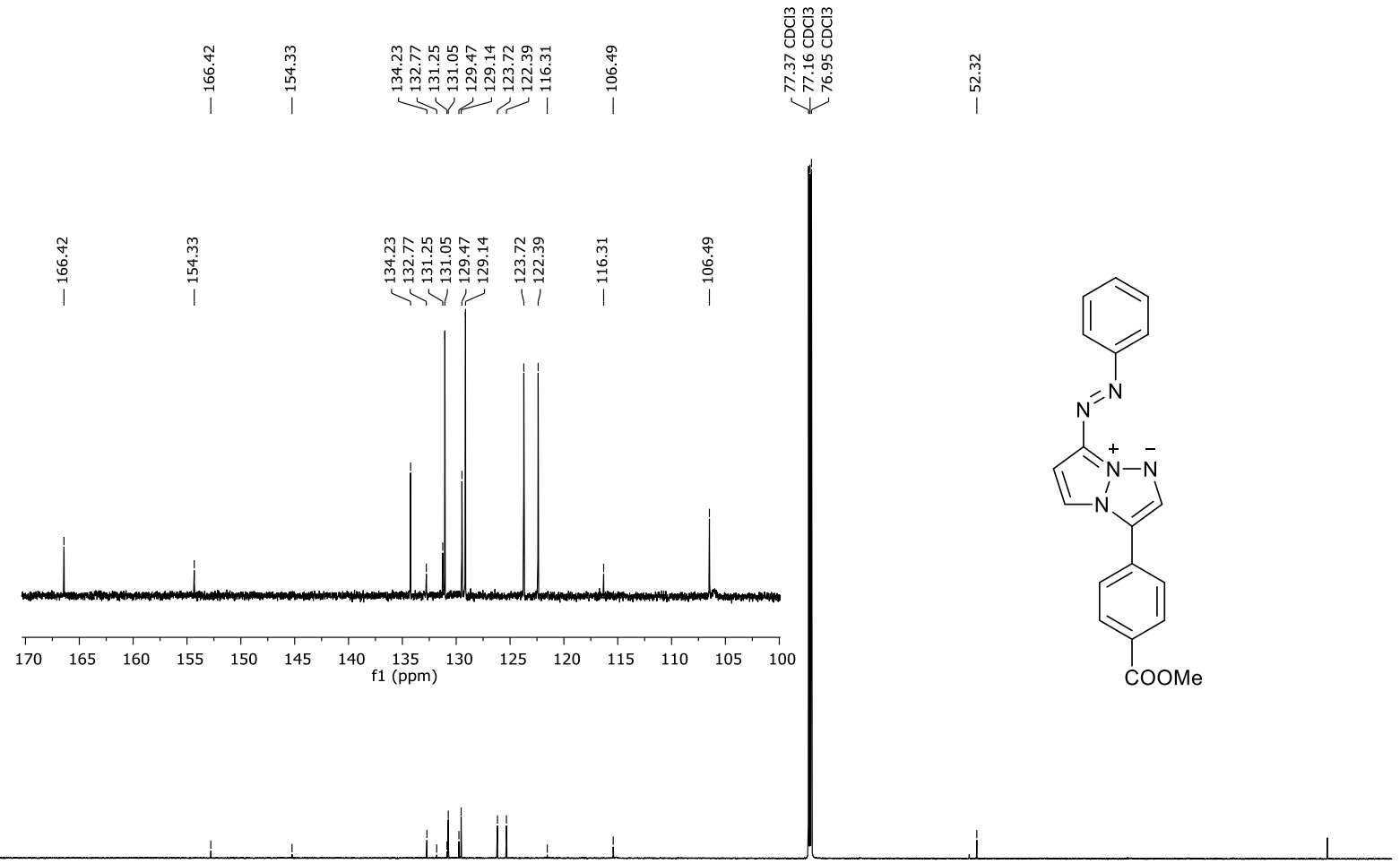

$\begin{array}{llllllllll}190 & 180 & 170 & 160 & 150 & 140 & 130 & 120 & 110 & 100 \\ \mathrm{f} 1(\mathrm{ppm})\end{array}$ 
TAP3, ${ }^{1} \mathrm{H}, 600 \mathrm{MHz}, \mathrm{CDCl}_{3}$

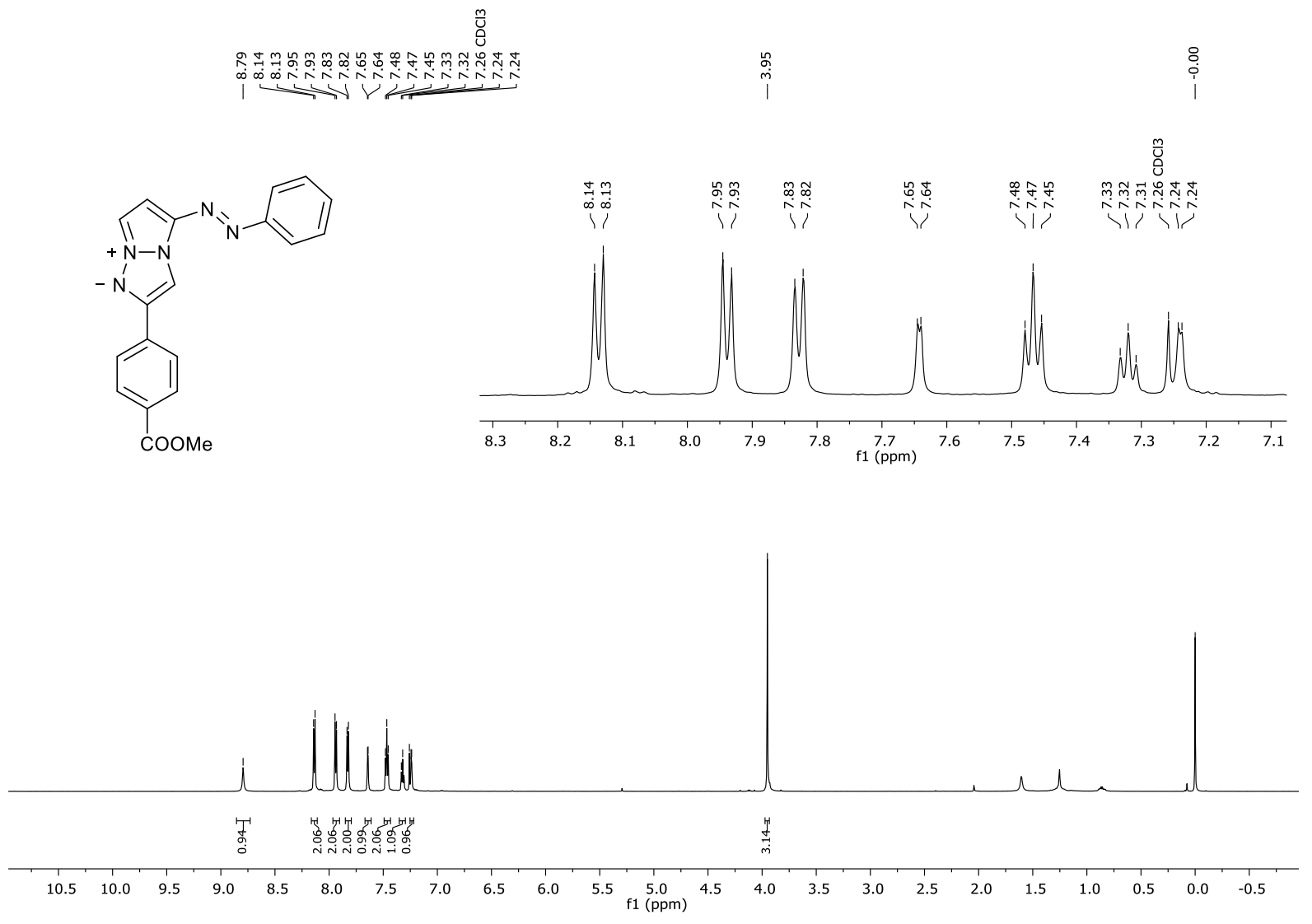

TAP3, ${ }^{13} \mathrm{C}\left\{{ }^{1} \mathrm{H}\right\}, 151 \mathrm{MHz}, \mathrm{CDCl}_{3}$

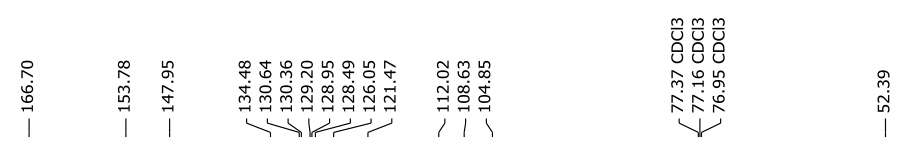

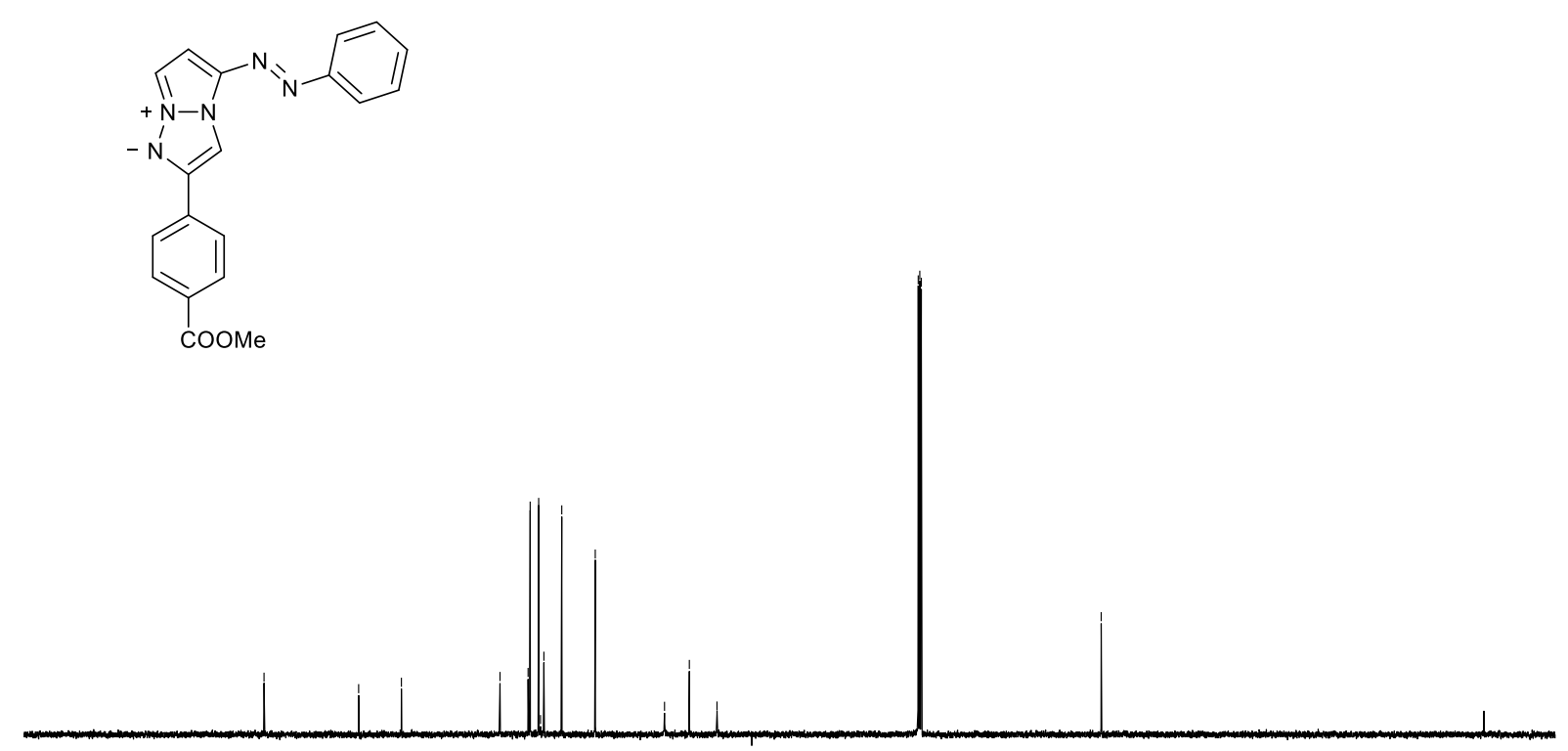

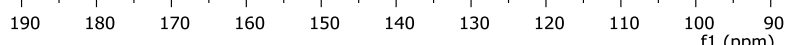


TAP4, ${ }^{1} \mathrm{H}, 400 \mathrm{MHz}, \mathrm{CDCl}_{3}$

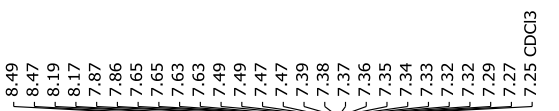
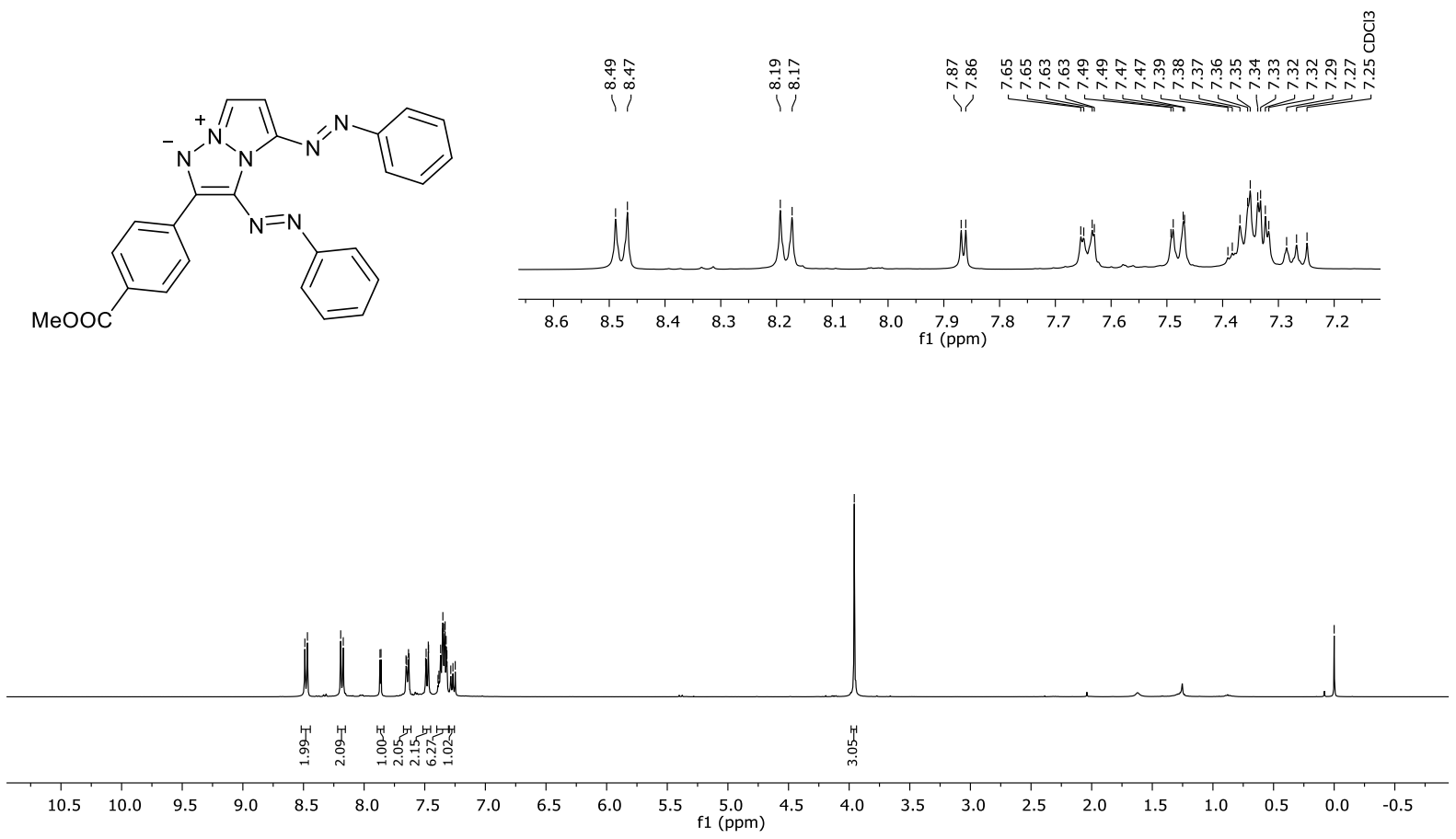

TAP4, ${ }^{13} \mathrm{C}\left\{{ }^{1} \mathrm{H}\right\}, 101 \mathrm{MHz}, \mathrm{CDCl}_{3}$

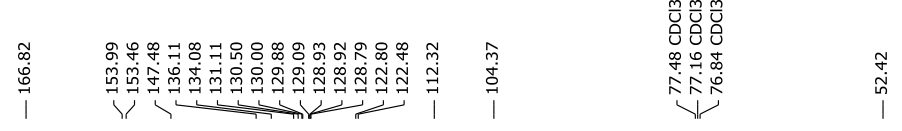<smiles></smiles>

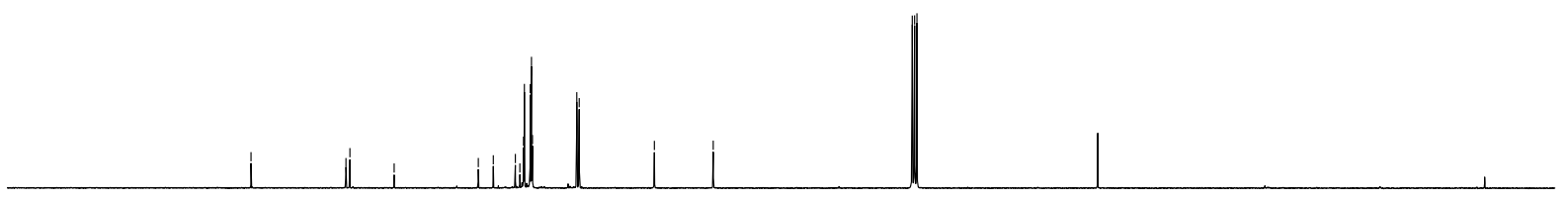

$\begin{array}{llllllllll}190 & 180 & 170 & 160 & 150 & 140 & 130 & 120 & 110 & \begin{array}{c}100 \\ \mathrm{f} 1(\mathrm{ppm})\end{array}\end{array}$ 
3, ${ }^{1} \mathrm{H}, 300 \mathrm{MHz}, \mathrm{DMSO}-d_{6}$<smiles>c1ccc(/N=N/c2ccn[nH]2)cc1</smiles>

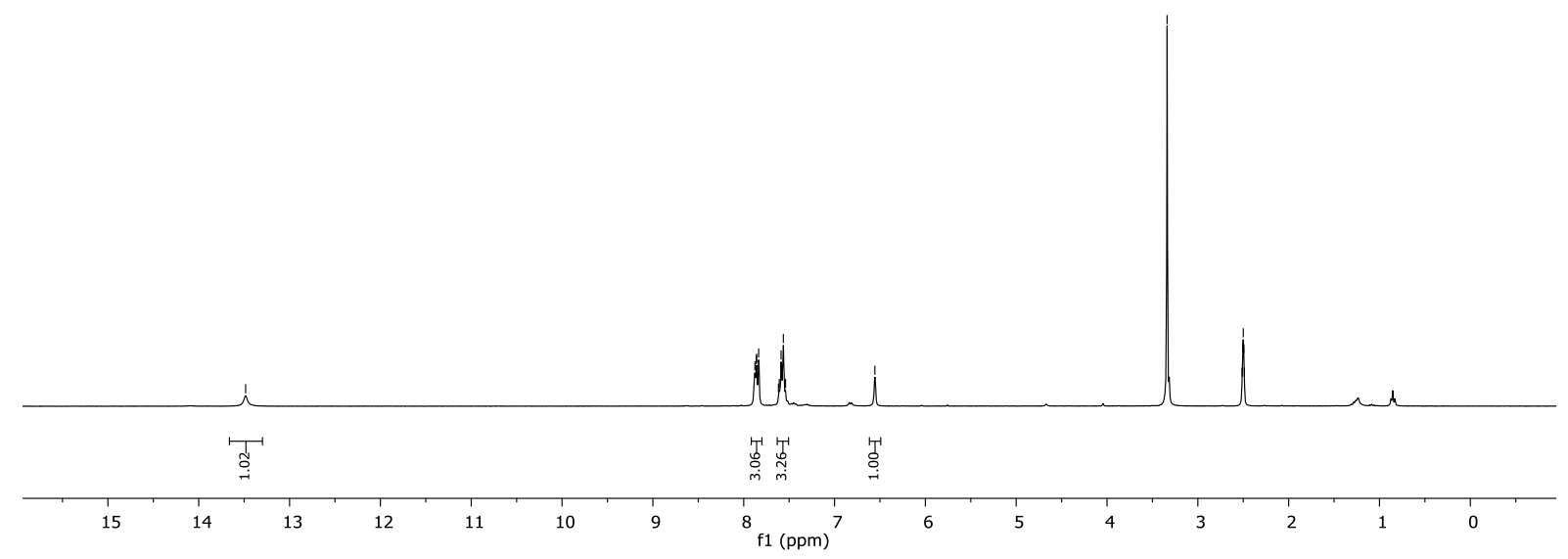

3, ${ }^{13} \mathrm{C}\left\{{ }^{1} \mathrm{H}\right\}, 75 \mathrm{MHz}$, DMSO-d

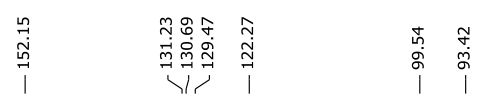
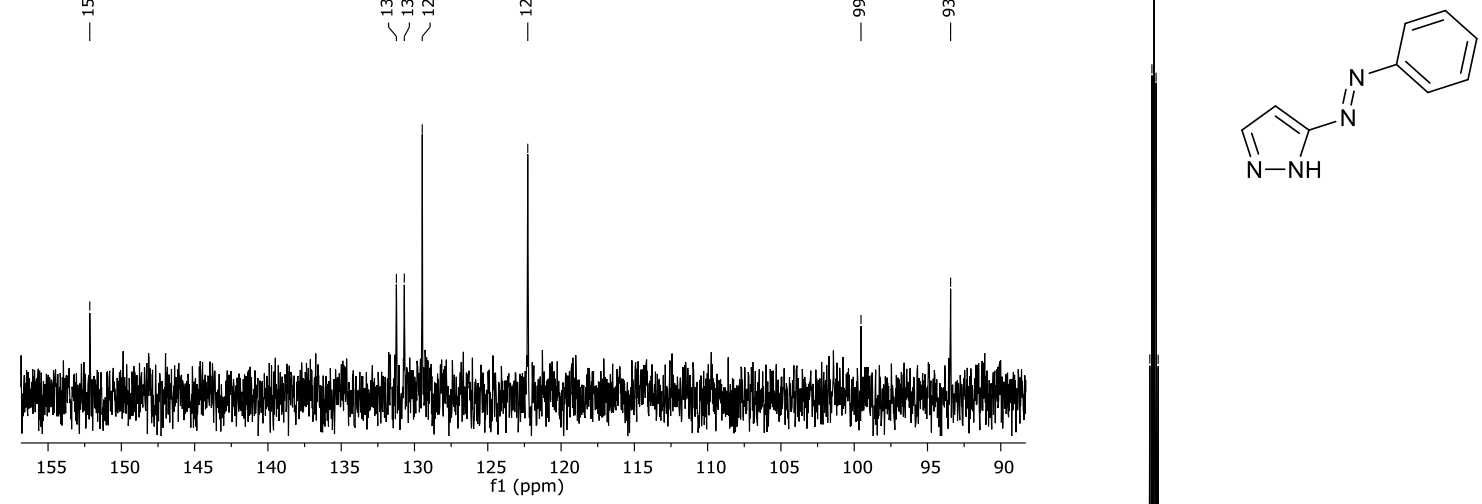

$\begin{array}{llllllllll}190 & 180 & 170 & 160 & 150 & 140 & 130 & 120 & 110 & 100 \\ \mathrm{f} 1(\mathrm{ppm})\end{array}$ 
4, ${ }^{1} \mathrm{H}, 300 \mathrm{MHz}$, DMSO- $d_{6}$

i<smiles>CC(=O)c1ccc(-c2cn[nH]n2)cc1</smiles>

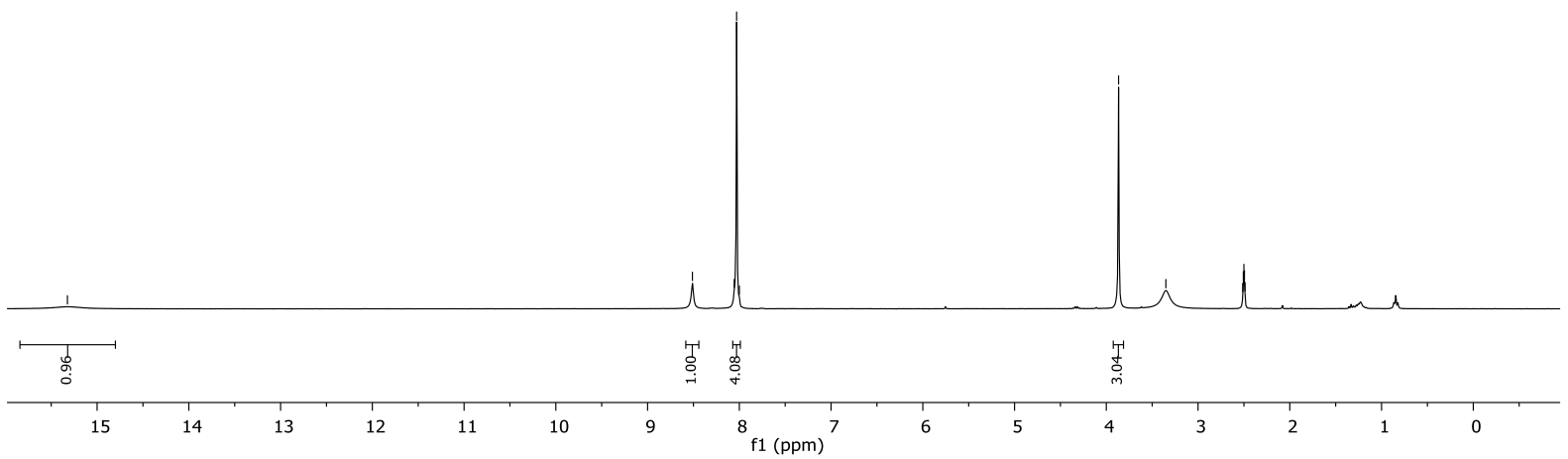

4, ${ }^{13} \mathrm{C}\left\{{ }^{1} \mathrm{H}\right\}, 75 \mathrm{MHz}$, DMSO- $d_{6}$

\begin{tabular}{|c|}
\hline \\
\hline \\
\hline \\
\hline \\
\hline \\
\hline \\
\hline 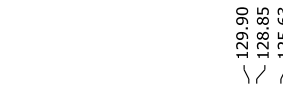 \\
\hline
\end{tabular}<smiles>CC(=O)c1ccc(-c2cn[nH]n2)cc1</smiles>

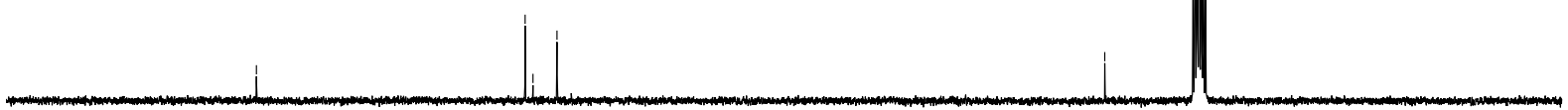


5, ${ }^{1} \mathrm{H}, 300 \mathrm{MHz}, \mathrm{CDCl}_{3}$

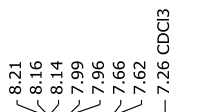

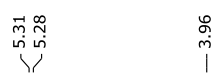

$\stackrel{8}{i}$<smiles>CC(=O)c1ccc(-c2cnn(/C=C\C#N)n2)cc1</smiles>

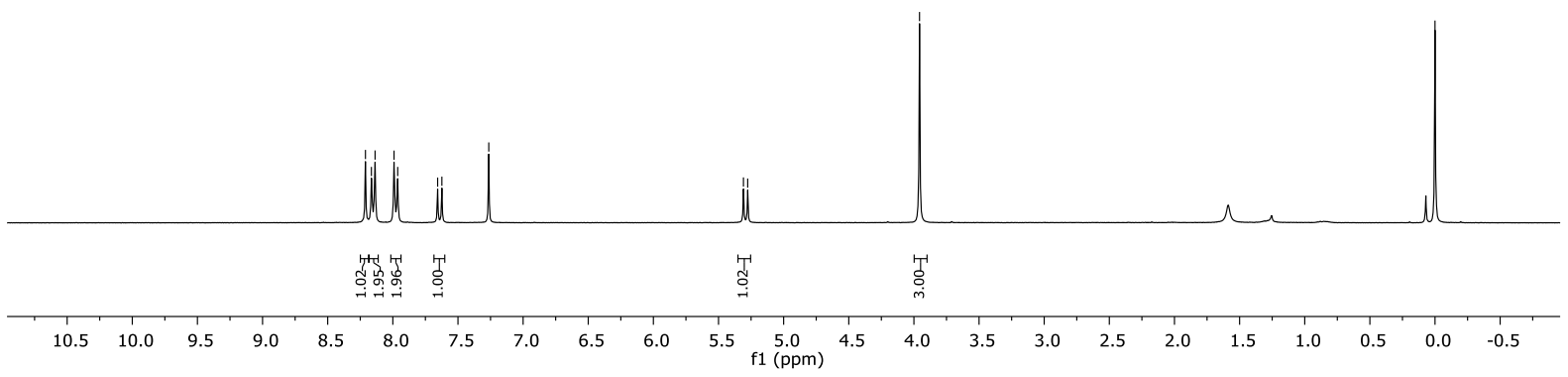

5, ${ }^{13} \mathrm{C}\left\{{ }^{1} \mathrm{H}\right\}, 75 \mathrm{MHz}, \mathrm{CDCl}_{3}$

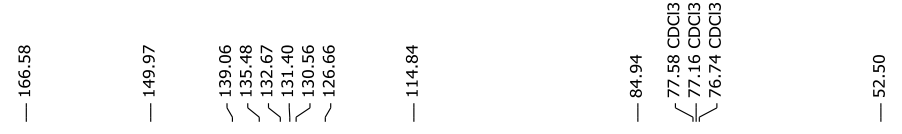<smiles>CC(=O)c1ccc(-c2cnn(/C=C\C#N)n2)cc1</smiles>

$\begin{array}{llllllllll}190 & 180 & 170 & 160 & 150 & 140 & 130 & 120 & 110 & 100 \\ \mathrm{f} 1(\mathrm{ppm})\end{array}$ 


\section{IR spectrum of 5}

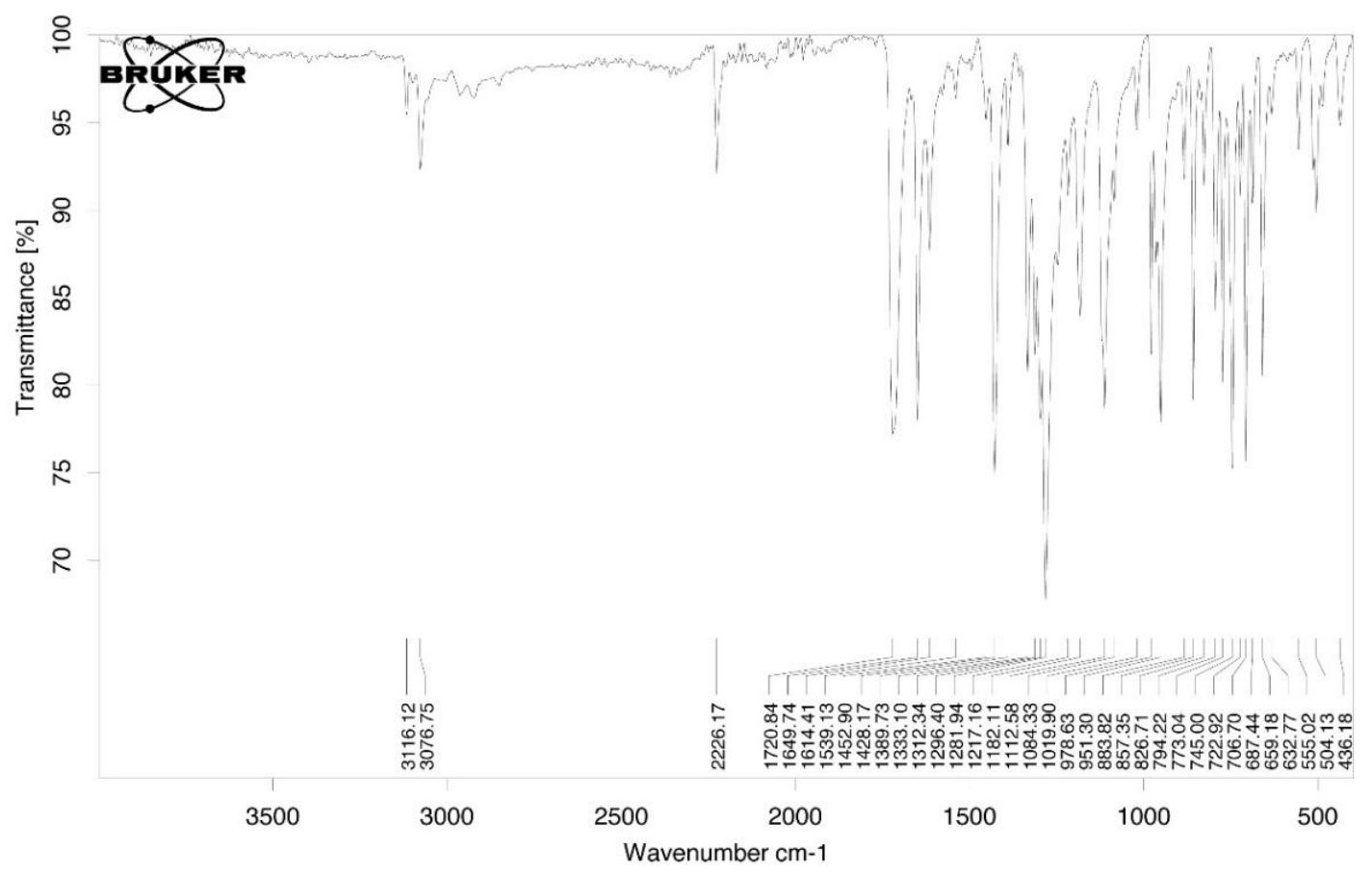

Figure S2. FTIR spectrum of methyl (Z)-4-(2-(2-cyanovinyl)-2H-1,2,3-triazol-4-yl)benzoate (5).

\section{UV-visible absorption spectra}

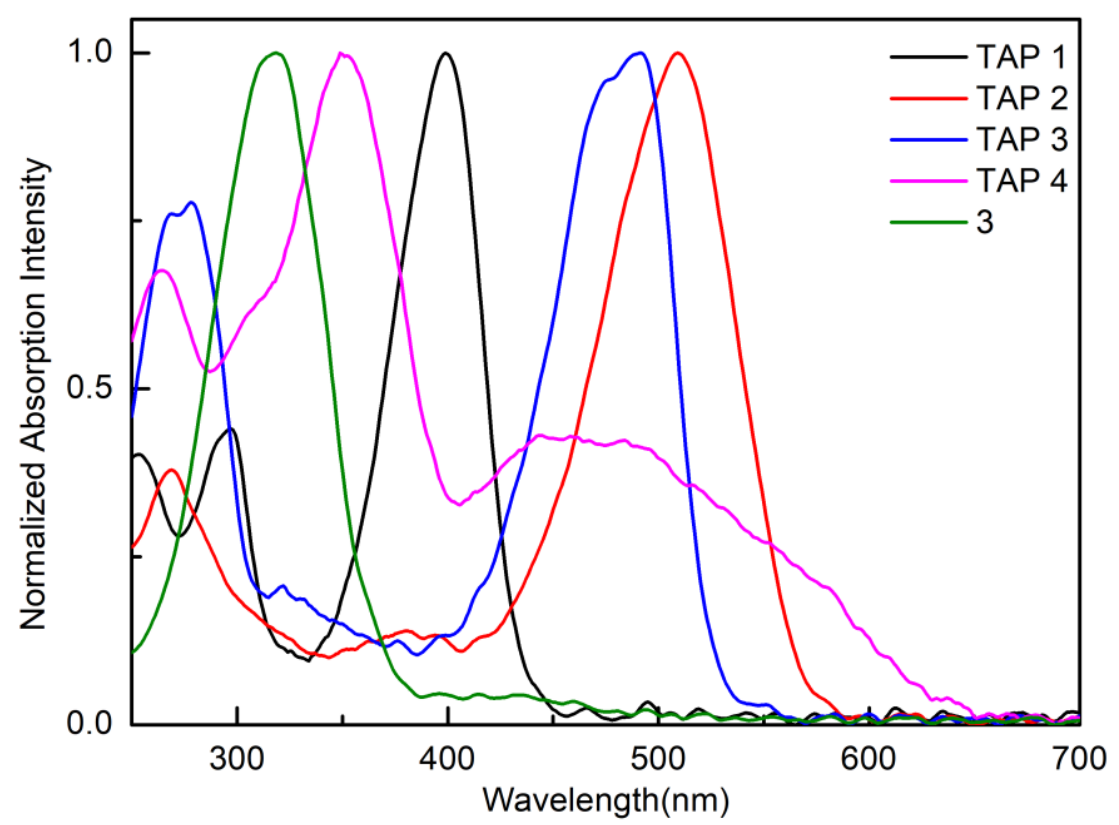

Figure S3. Normalized absorption spectra of TAP1-4 and $\mathbf{3}$ in solution in dichloromethane. 


\section{Crystallographic data}

\section{Crystal structure determination of TAP2}

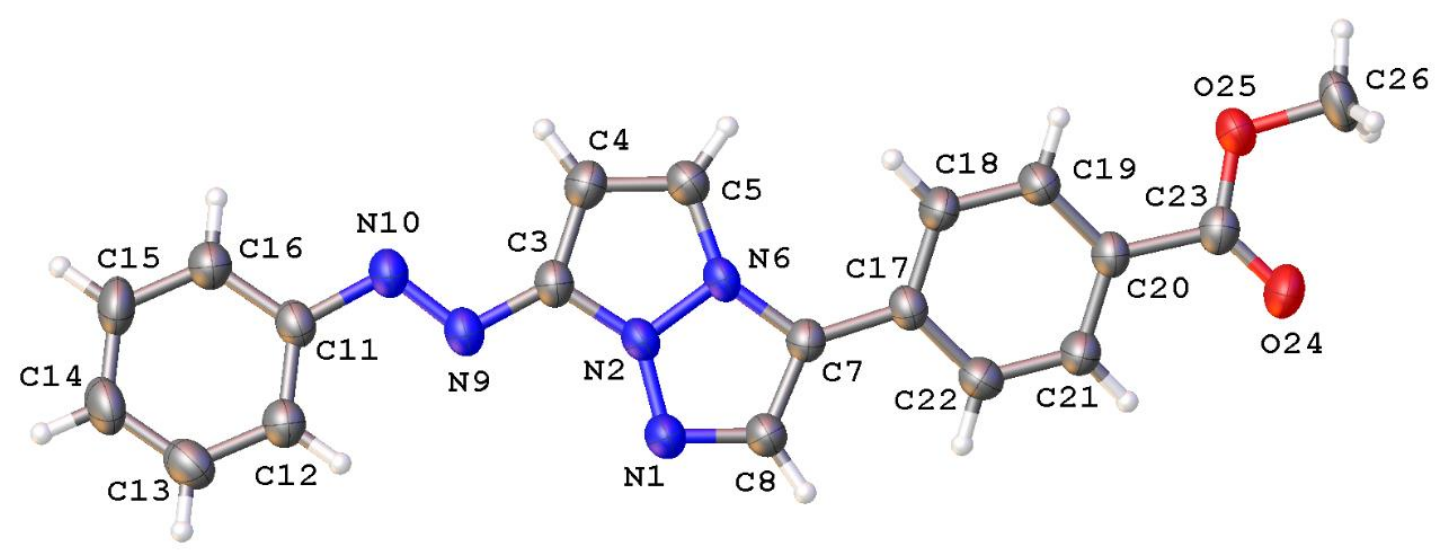

Figure S4. Crystal structure of TAP2. Thermal ellipsoids are drawn at the 50\% probability level.

\section{Experimental}

Single crystals of TAP2 were grown from a mixture of chloroform, ethanol and dichloromethane. A suitable crystal was selected and X-ray diffraction data were collected on an Agilent SuperNova diffractometer, equipped with an Eos CCD detector, using Mo Ka radiation $(\lambda=0.71073 \AA)$. The crystal was kept at 293(2) K during data collection. Using Olex $2,{ }^{1}$ the structure was solved with the SHELXT ${ }^{2}$ structure solution program using Intrinsic Phasing and refined with the SHELXL ${ }^{3}$ refinement package using full-matrix least squares minimization on $F^{2}$. CCDC 1995012 contains the supplementary crystallographic data for TAP2 and can be obtained free of charge via www.ccdc.cam.ac.uk/conts/retrieving.html (or from the Cambridge Crystallographic Data Centre, 12, Union Road, Cambridge CB2 1EZ, UK; fax: +44-1223-336033; or deposit@ccdc.cam.ac.uk).

\section{Table S1: Crystal data and structure refinement for TAP2.}

$\begin{array}{ll}\text { Empirical formula } & \mathrm{C}_{19} \mathrm{H}_{15} \mathrm{~N}_{5} \mathrm{O}_{2} \\ \text { Formula weight } & 345.36 \\ \text { Temperature/K } & 293(2) \\ \text { Crystal system } & \text { triclinic } \\ \text { Space group } & \mathrm{P}-1 \\ \mathrm{a} / \AA & 9.1471(6) \\ \mathrm{b} / \AA & 10.2389(12) \\ \mathrm{c} / \AA & 10.4577(9) \\ \alpha /{ }^{\circ} & 60.722(10) \\ \beta /{ }^{\circ} & 78.914(6) \\ \gamma /{ }^{\circ} & 71.326(8)\end{array}$


Volume/ $\AA^{3}$

Z

$\rho_{\text {calc }} \mathrm{g} / \mathrm{cm}^{3}$

$\mu / \mathrm{mm}^{-1}$

$\mathrm{F}(000)$

Crystal size $/ \mathrm{mm}^{3}$

Radiation

$2 \Theta$ range for data collection $/^{\circ}$

Index ranges

Reflections collected

Independent reflections

Data/restraints/parameters

Goodness-of-fit on $\mathrm{F}^{2}$

Final R indexes $[\mathrm{I}>=2 \sigma(\mathrm{I})]$

Final $R$ indexes [all data]

Largest diff. peak/hole / e $\AA^{-3}$
$808.58(15)$

2

1.418

0.097

360.0

$0.5 \times 0.2 \times 0.05$

$\operatorname{Mo} \mathrm{K} \alpha(\lambda=0.71073)$

4.786 to 52.734

$-11 \leq \mathrm{h} \leq 11,-12 \leq \mathrm{k} \leq 12,-13 \leq 1 \leq 13$

11341

$3299\left[\mathrm{R}_{\text {int }}=0.0561, \mathrm{R}_{\text {sigma }}=0.0573\right]$

$3299 / 0 / 236$

1.066

$\mathrm{R}_{1}=0.0690, \mathrm{wR}_{2}=0.1747$

$\mathrm{R}_{1}=0.1115, \mathrm{wR}_{2}=0.2037$

$0.27 /-0.24$

\section{Crystal structure determination of TAP3}

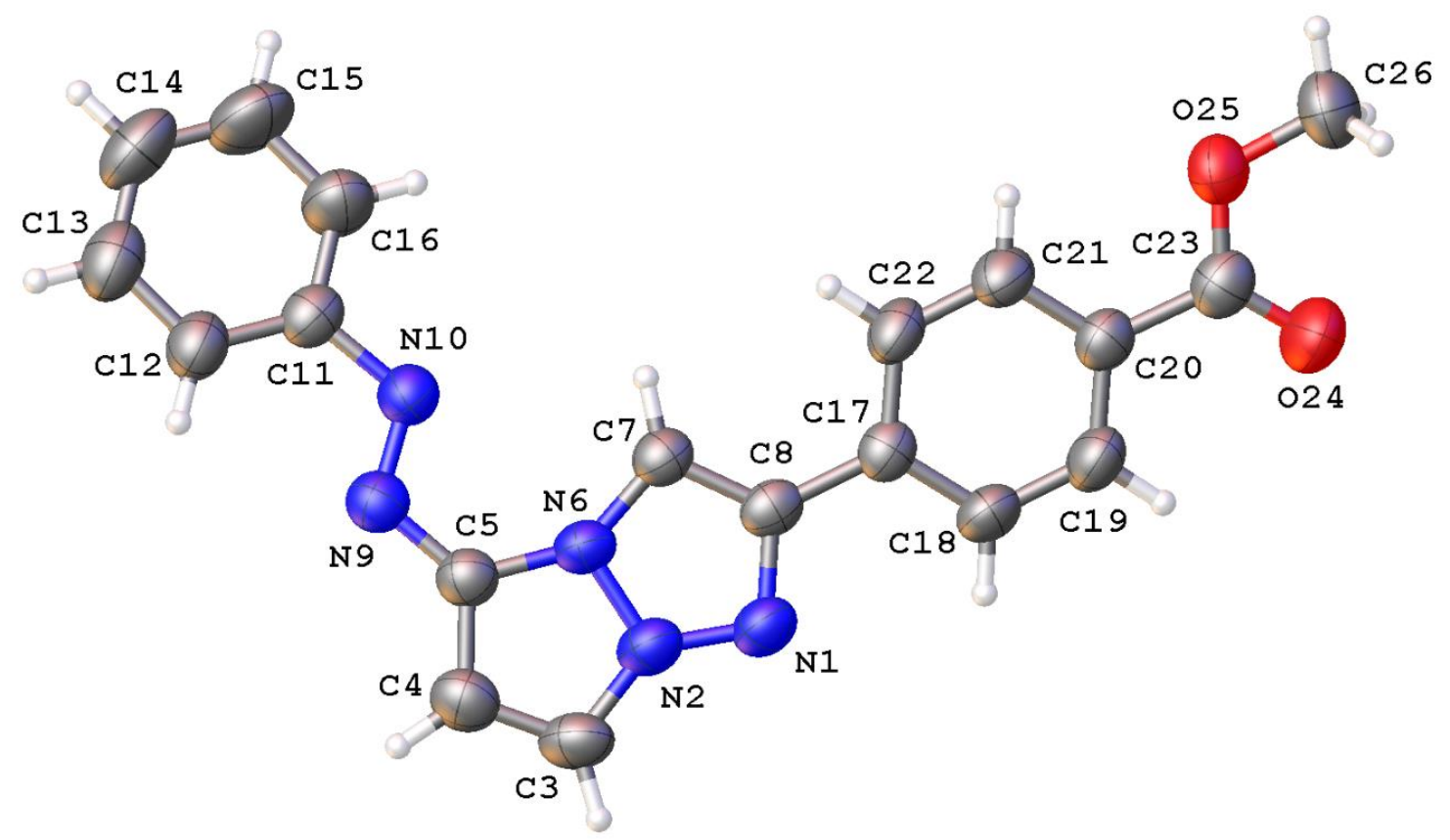

Figure S5. Crystal structure of TAP3. Thermal ellipsoids are drawn at the 50\% probability level. The asymmetric unit contains three molecules, only one molecule is shown. 


\section{Experimental}

Single crystals of TAP3 were grown from ethanol. A suitable crystal was selected and X-ray diffraction data were collected on an Agilent SuperNova diffractometer, equipped with an Eos CCD detector, using Mo Ka radiation ( $\lambda=0.71073 \AA$ ). The crystal was kept at $293(2) \mathrm{K}$ during data collection. Using Olex $2,{ }^{1}$ the structure was solved with the ShelXS ${ }^{4}$ structure solution program using Direct Methods and refined with the ShelX $\mathrm{L}^{3}$ refinement package using full-matrix least squares minimization on $F^{2}$. The asymmetric unit contains three molecules. CCDC 1995011 contains the supplementary crystallographic data for TAP3 and can be obtained free of charge via www.ccdc.cam.ac.uk/conts/retrieving.html (or from the Cambridge Crystallographic Data Centre, 12, Union Road, Cambridge CB2 1EZ, UK; fax: +44-1223-336033; or deposit@ccdc.cam.ac.uk).

\section{Table S2: Crystal data and structure refinement for TAP3.}

\begin{tabular}{|c|c|}
\hline Empirical formula & $\mathrm{C}_{19} \mathrm{H}_{15} \mathrm{~N}_{5} \mathrm{O}_{2}$ \\
\hline Formula weight & 345.36 \\
\hline Temperature/K & 293(2) \\
\hline Crystal system & triclinic \\
\hline Space group & $\mathrm{P}-1$ \\
\hline $\mathrm{a} / \AA$ & $10.4950(6)$ \\
\hline $\mathrm{b} / \AA$ & $14.6071(6)$ \\
\hline$c / \AA$ & $17.6793(6)$ \\
\hline$\alpha /^{\circ}$ & $71.132(3)$ \\
\hline$\beta /{ }^{\circ}$ & $83.894(4)$ \\
\hline$\gamma /{ }^{\circ}$ & $76.917(4)$ \\
\hline Volume $/ \AA^{3}$ & $2496.4(2)$ \\
\hline $\mathrm{Z}$ & 6 \\
\hline$\rho_{\text {calc }} \mathrm{g} / \mathrm{cm}^{3}$ & 1.378 \\
\hline$\mu / \mathrm{mm}^{-1}$ & 0.094 \\
\hline $\mathrm{F}(000)$ & 1080.0 \\
\hline Crystal size/mm $\mathrm{mm}^{3}$ & $0.5 \times 0.15 \times 0.05$ \\
\hline Radiation & $\operatorname{MoK} \alpha(\lambda=0.71073)$ \\
\hline $2 \Theta$ range for data collection $/{ }^{\circ}$ & 4.872 to 52.74 \\
\hline Index ranges & $-13 \leq \mathrm{h} \leq 13,-18 \leq \mathrm{k} \leq 18,-22 \leq 1 \leq 22$ \\
\hline Reflections collected & 51091 \\
\hline Independent reflections & $10167\left[R_{\text {int }}=0.0353, R_{\text {sigma }}=0.0370\right]$ \\
\hline Data/restraints/parameters & 10167/0/706 \\
\hline Goodness-of-fit on $\mathrm{F}^{2}$ & 1.023 \\
\hline Final $R$ indexes $[I>=2 \sigma(I)]$ & $\mathrm{R}_{1}=0.0792, \mathrm{wR}_{2}=0.2122$ \\
\hline Final $\mathrm{R}$ indexes [all data] & $\mathrm{R}_{1}=0.1364, \mathrm{wR}_{2}=0.2607$ \\
\hline Largest diff. peak/hole / e $\AA^{-3}$ & $0.43 /-0.24$ \\
\hline
\end{tabular}




\section{References}

1 O. V Dolomanov, L. J. Bourhis, R. J. Gildea, J. A. K. Howard and H. Puschmann, J. Appl. Crystallogr., 2009, 42, 339-341.

$2 \quad$ G. Sheldrick, Acta Crystallogr. Sect. A, 2015, 71, 3-8.

3 G. Sheldrick, Acta Crystallogr. Sect. C, 2015, 71, 3-8.

$4 \quad$ G. Sheldrick, Acta Crystallogr. Sect. A, 2008, 64, 112-122. 